\title{
Optical Microfiber Sensors for the Detection of Current Pulses
}

\author{
George Y. Chen, Timothy Lee, Rand Ismaeel, Mohammad Belal, Trevor P. Newson and Gilberto Brambilla* \\ Optoelectronics Research Centre, University of Southampton, Southampton SO17 1BJ, United Kingdom \\ Author e-mail address:gb2@orc.soton.ac.uk
}

\begin{abstract}
Issues and benefits of using optical microfibers for current sensing are discussed. OCIS codes: (060.2370) Fiber optics sensors; (230.2240) Faraday effect.
\end{abstract}

\section{Introduction}

Current sensors exploiting the Faraday Effect in optical fibers [1] have attracted considerable interest because of their robustness and configurability, thus compatibility with remote sensing. Yet, in conventional optical fibers the minimization of bending losses results in a large sensor size (typically in excess of $\sim 10 \mathrm{~cm}$ ). Moreover, as optical fibers exhibit small responsivities to currents, long lengths of fiber have to be deployed in a single sensor. Since the sensor response time is related to the light time of flight into the sensing device, long lengths of fiber are associated with response times longer than $\mu \mathrm{s}$.

Since 2003, when Tong and co-workers demonstrated that optical microfibers (OMs) can have low propagation losses [2] and therefore can be used to manufactured devices, OMs have been proposed for a wealth of sensing applications [3]. Because of their reduced size, OM coils [4] and coil resonators [5] have found application in current sensing, with $\mathrm{mm}$ size sensors being demonstrated. In this paper the use of OMs for current sensing is reviewed, with an analysis of its benefits and potential issues.

\section{Faraday Effect in OM coils}

When linearly polarized light propagates in a medium in an electromagnetic field, its polarization azimuth experiences a rotation $\theta$ proportional to the Verdet constant $V$ of the material, to the magnetic field $B$ and to the distance $L$ over which this interaction occurs. In the case of an OM coil with $N$ turns wrapped around a current wire, the polarization azimuth can then be expressed as:

$$
\theta=B V L=(\mu I / 2 \pi r) \cdot V \cdot N 2 \pi r=N \mu I V
$$

where $I$ and $\mu$ are the current and the medium magnetic permeability $\left(=\mu_{0} \mu_{r}\right.$, where $\mu_{0}$ is the vacuum magnetic permeability and $\mu_{r}$ the medium relative permeability), respectively. $L$ is approximated by the microfiber length. Eq. (1) shows that for given $I$ and fiber (thus $V$ ), high $\theta$ can be achieved increasing the number of turns in the OM coil. Still, this affects the device bandwidth and, more importantly its response at high frequencies. Increasing $N$ decreases the maximum measurable frequency.

OM coils are particularly attractive because they have the possibility to measure single current pulses. The minimum current pulse duration measurable by this type of sensor is related to the light time of flight $(t)$ in the sensor, which can be approximated by:

$$
t=L /(\mathrm{c} / n)=2 \pi r N n / \mathrm{c}
$$

where $c$ is the speed of light in vacuum. A sensor with $N=25, r=0.5 \mathrm{~mm}$ and $n=1.45$ (telecom optical fiber) has $t \sim 0.38 \mathrm{~ns}$, thus it can sense current pulses of the order of ns. For comparison, current sensors based on optical fibers usually sense pulses considerably longer than $1 \mu \mathrm{s}$.

\section{A linear sensor}

Fig. 1(a) shows the OM sensor head used to measure ns current pulses: linearly polarized light from a laser diode was injected into the sensor and its polarization state at the sensor output was analyzed with a polarizer in quadrature and a powermeter. Fig. 1(b) shows the response time of the OM sensor: the optical response follows the current temporal evolution to a sub-ns precision.

The sensor minimum detectable current is limited by the minimum measurable azimuth rotation and for the set-up used in these preliminary experiments currents as low as $40 \mathrm{~mA}$ could be measured. The measurement of smaller currents with the same set-up requires a larger $N$, and thus $L$. Yet, the manufacture and handling of long tapers with 
small diameter (typically of the order of magnitude of the wavelength propagating in it) is extremely challenging and from the practical point of view it is limited to few centimeters.
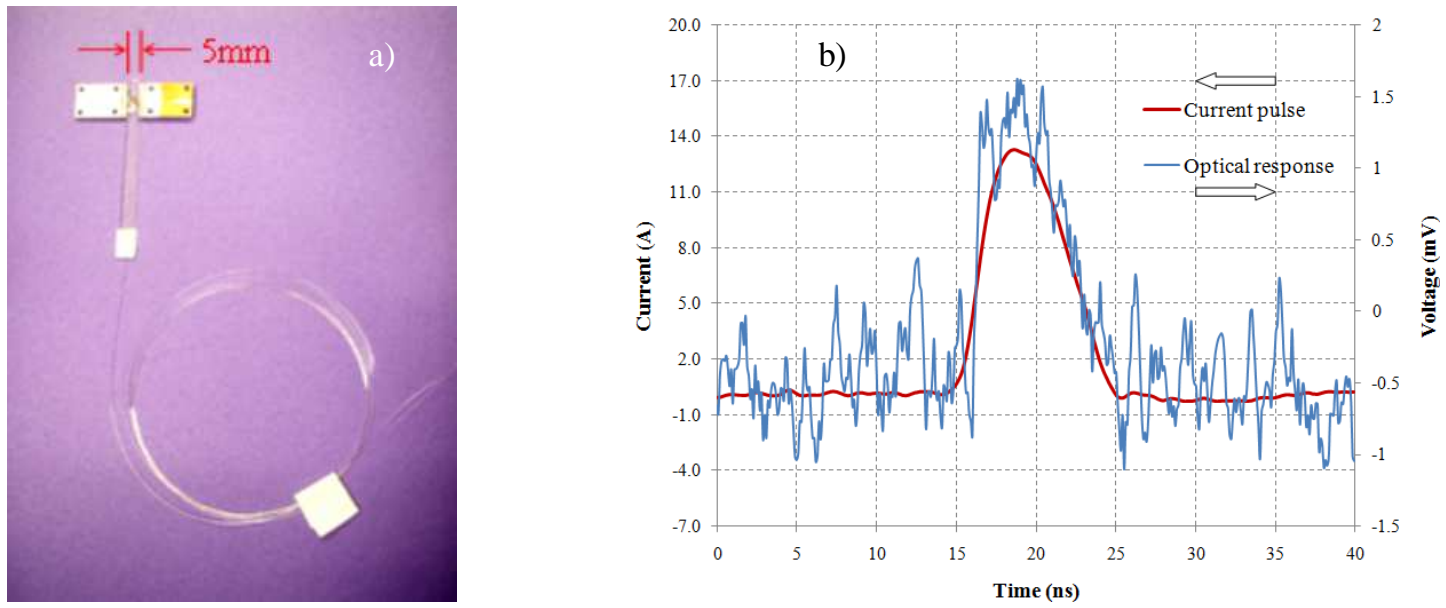

Fig. 1 (a) Picture of an OM current sensor made from a coil with 25 turns. (b) Comparison between the temporal evolution of a current pulse injected in the OM sensor and the sensor optical response. Rising time is smaller than $1 \mathrm{~ns}$.

\section{A resonant sensor}

An alternative method to increase the minimum measurable current relies on the sole increase of the optical path length, achieved by using OM resonators. OM coil resonators exploit the evanescent field of the mode propagating inside the OM to couple light between different loops. Because of the light recirculation at resonance, coil resonators effectively increase the optical path without increasing the overall OM physical length.

The resonant sensor design is more complex than the linear one (Fig. 2): linearly polarized light from a tunable laser source is launched into a polarization-maintaining fiber, where a $45^{\circ}$ splice splits power equally in two orthogonal polarizations. Light propagates through the polarization-maintaining coupler and a polarization controller before reaching the OM coil resonator sensor. The Faraday rotator mirror reflects light propagating along each axis via the other axis and it is used to compensate for fluctuations in state of polarization along the OM coil resonator. The PM fiber translates the signal into an intensity modulation, which is then detected by using a polarization beamsplitter to separate the two polarizations in the two photodiodes of a balanced detector. This is aimed at reducing noise and amplifying the difference between the two polarization amplitudes.

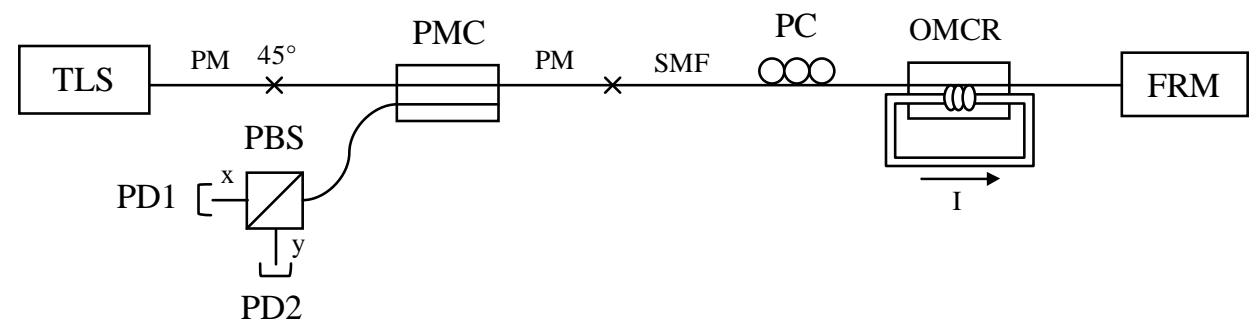

Fig. 2 Schematic of the resonant version of the OM current sensor. Light is launched from a tunable laser source (TLS) into a polarizationmaintaining (PM) fiber. A polarization-maintaining coupler (PMC) is spliced at $45^{\circ}$ to split power between the two different polarizations. A polarization controller (PC) is used before the optical microfiber coil resonator (OMCR) and the Faraday rotator mirror (FRM). Reflected light is then split by a polarization beam-splitter (PBS) and the two polarizations detected by a balanced detector (PD1 and PD2).

The device response for a 3-turn OM coil resonator manufactured from a $2 \mu \mathrm{m}$ diameter OM is presented in fig. 3(a). The OM was $10 \mathrm{~mm}$ long and the coil diameter was $\mathrm{d} \sim 1 \mathrm{~mm}$ (a thin layer of low-refractive-index, UV-curable polymer was deposited on a $1 \mathrm{~mm}$ diameter metallic rod before the OM was coiled). The sensor showed at resonance an average responsivity of $4.4 \mu \mathrm{rad} / \mathrm{A}$, which was 3.1 times larger than that observed off resonance. Fig. 3(b) shows the calculated responsivity enhancement factor for different resonance strengths. Enhancement has a 
nonlinear dependence on the resonance strength with stronger resonances experiencing a considerably higher enhancement. For a $10.9 \mathrm{~dB}$ resonance strength, fig. 2(c) predicts a 3.25 times enhancement, comparable to that observed experimentally.
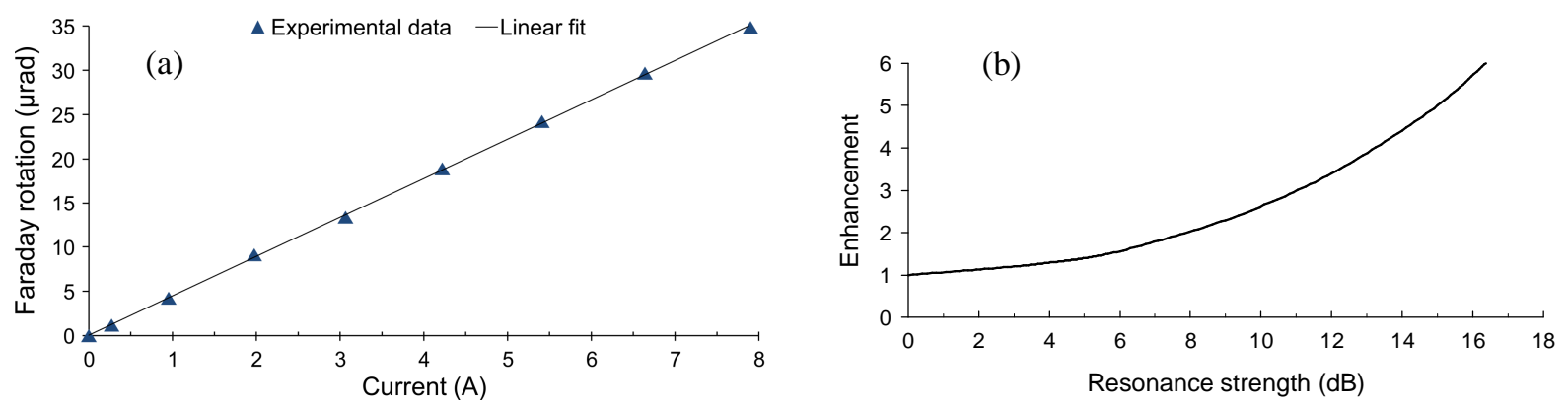

Fig. 3 (a) Optical response of the OM coil resonant sensor shown in fig. 2. The current signal was modulated at 7.9 A, $30 \mathrm{~Hz}$. The OM coil resonator had $10.9 \mathrm{~dB}$ deep resonances at $\lambda \sim 1.5 \mu \mathrm{m}$. (b) Optical response enhancement in the resonant sensor with respect to the linear sensor for different resonance strengths.

\section{Issues and opportunities}

Linear birefringence (LB) is present in all microfibers and because of the device geometry can always be observed in OM coil sensors. Because of the small nature of Faraday rotation in silica, even when present in small amounts, LB significantly reduces the effect of Faraday rotation. In fact, the Faraday rotation transfers power from one polarization to the other. If LB is not present, this contribution increases along the whole sensor length. On the contrary, when LB is not negligible, the Faraday contribution between the two axes reverses with a periodicity of half of the beat length as the relative phase increases along the propagation distance. In optical fibers issues related to LB have been overcome by using spun fibers [6], which involve preform spinning during fiber drawing to average LB between fast and slow axes. Spun microfibers are also being proposed [7] to reduce this effect in OM.

The sensor sensitivity is ultimately determined by the material used, as the Verdet constant is material dependent and for silica is relatively small $(\mathrm{V}=0.54 \mathrm{rad} / \mathrm{T} \cdot \mathrm{m})$. Optical fibers doped with transition elements have shown a large increase in $V[8,9]$. The manufacture of OMs from materials with high $V$ would increase the current sensor responsivity without compromising its bandwidth/response time. Sensing of current pulses smaller than $1 \mathrm{~mA}$ can be envisaged.

\section{References}

[1] G. W. Day, D. N. Payne, A. J. Barlow, and J. J. Ramskov-Hansent, "Faraday rotation in coiled, monomode optical fibers: isolators, filters, and magnetic sensors", Opt. Lett. 7, 238-240 (1982).

[2] L. M. Tong, R.R. Gattass, J.B. Ashcom, S.L. He, J.Y. Lou, M.Y. Shen, I. Maxwell, and E. Mazur, "Subwavelength-diameter silica wires for low-loss optical wave guiding" Nature 426 816-819 (2003).

[3] G. Brambilla, “Optical fibre nanotaper sensors”, Optical Fiber Technology 16, 331-342 (2010).

[4] M. Belal, Z. Song, Y. Jung, G. Brambilla, and T. P. Newson, “Optical fiber microwire current sensor”, Opt. Lett. 35, $3045-3047$ (2010).

[5] G. Y. Chen, T. Lee, R. Ismaeel, G. Brambilla, and T. P. Newson, "Resonantly enhanced Faraday rotation in a microcoil current sensor", IEEE Photon. Technol. Lett. DOI: 10.1109/LPT.2012.2189381 in press.

[6] A. J. Barlow, J. J. Ramskov-Hansen, and D. N. Payne, "Birefringence and polarization mode-dispersion in spun single-mode fibers", Appl. Optics 20(17), 2962-2968 (1981).

[7] G. Y. Chen, G. Brambilla, and T. P. Newson, “Spun optical microfiber”, submitted.

[8] J. Ballato and E. Snitzer, "Fabrication of fibers with high rare-earth concentrations for Faraday isolator applications," Appl. Opt. 34, 6848$6854(1995)$

[9] L. Sun, S. Jiang, and J. R. Marciante, "Compact all-fiber optical Faraday components using 65-wt\%-terbium-doped fiber with a record Verdet constant of -32 rad/(Tm)," Opt. Express 18, 12191-12196 (2010) 